ISSN 1991-8631

Original Paper

http://indexmedicus.afro.who.int

\title{
La production du miel à Manigri (Commune de Bassila) au Bénin: enjeu et importance socio-économique
}

\author{
Hounnankpon YÉDOMONHAN * et Akpovi AKOÈGNINOU \\ Laboratoire de Botanique et d'Ecologie Végétale, Faculté des Sciences et Techniques, Université \\ d'Abomey-Calavi, 01 BP 4521 Cotonou, Bénin. \\ *Auteur correspondant, E-mail : h.yedo@yahoo.fr ; Tél. (00229)95564054
}

\section{RESUME}

Trente cinq apiculteurs dont l'âge est compris entre 30 et 79 ans ont été enquêtés sur la production et les vertus du miel. Soixante dix pour-cent (70\%) d'eux pratiquaient la chasse au miel avant d'être convertis en apiculteurs modernes. Le nombre de ruches par apiculteur varie de 3 à 38. La production annuelle en miel est en moyenne de 11,1 \pm 3,7 litres par ruche et de 125,2 \pm 95,7 litres par apiculteur. Le prix de vente du miel varie entre 1000 et $1500 \mathrm{~F} \mathrm{CFA}$ le litre. Le revenu annuel brut par apiculteur se situe entre 24300 à 670320 FCFA. Les charges liées à l'équipement d'installation ne permettent pas à tous les apiculteurs d'amortir leurs investissements à la première année d'activité. Le miel est utilisé dans le traitement médico-magique de 20 maladies. La toux et la fièvre sont les plus usuelles.

(C) 2009 International Formulae Group. All rights reserved.

Mots clés : Miel, production, revenus annuels, vertus, Manigri.

\section{INTRODUCTION}

L'apiculture est l'élevage des abeilles pour la production de miel. C'est une activité séculaire dans les pays de l'Afrique de l'Ouest (Villières, 1987). La production de miel a évolué de la chasse au miel à l'apiculture traditionnelle avec des ruches traditionnelles puis à l'apiculture moderne. La chasse au miel ou apiculture dite de cueillette consiste à repérer de jour des colonies d'abeilles dans des trous de gros arbres ou entre des rochers, et de récolter la nuit après destruction par le feu de la colonie d'abeilles. L'apiculture traditionnelle diffère de la précédente par l'utilisation de pièges à abeilles qui sont des ruches construites en matériaux locaux (troncs d'arbre évidés, paille, pot de terre cuite, etc.) et par l'utilisation de feu pour la récolte du miel, ce qui entraîne aussi la destruction des colonies d'abeilles et souvent des feux de végétation. L'apiculture moderne utilise non seulement des ruches améliorées mais aussi des techniques appropriées permettant de récolter le miel tout en conservant la colonie d'abeilles. Elle est une activité qui sollicite de faible main d'œuvre et offre la possibilité de l'associer à d'autres activités agricoles.

L'apiculture au Bénin est restée traditionnelle avec la chasse au miel et l'utilisation des ruches traditionnelles jusqu'en 1972 où l'apiculture moderne a vu le jour par Potiron (Hussein, 2001). Mais, ce n'est qu'en 1994 que le Centre Intégré du Bénin pour l'apiculture tropicale a été créé à Parakou pour organiser des stages de formation en apiculture et contribuer à la reconstitution des populations d'abeilles gravement affectées par la chasse au miel. Ainsi, une conscience collective sur l'apiculture moderne est apparue grâce à l'amélioration du revenu monétaire des 
acteurs et à l'utilisation des produits de la ruche en alimentation et en médecine.

Depuis quelques années, un regain d'intérêts est observé pour les Produits Forestiers Non Ligneux (PFNL) dont le miel (Anonyme, 2002). Leur contribution à l'économie des ménages, à la sécurité alimentaire et à certains objectifs écologiques tels que la conservation de la diversité biologique est très importante. La plupart des PFNL sont vendus dans les marchés locaux et régionaux. Leur commerce se chiffre à plusieurs millions de francs CFA par an. Pourtant, le Bénin, à l'instar de beaucoup d'autres pays africains ne dispose pas de données statistiques sur la filière apicole (Anonyme, 2005). Les paramètres comme le nombre d'apiculteurs, le nombre de colonies par apiculteur, la production totale annuelle du miel ainsi que son commerce et sa consommation ne sont pas connus. Ceci entrave la promotion de la filière. En effet, d'après Villières (1987), toute intervention dans le domaine apicole doit être précédée d'une étude du milieu comprenant les potentialités techniques de la zone, les facteurs climatiques de tout ordre, l'importance de l'insertion de l'apiculture dans le secteur agricole, les circuits commerciaux des produits de la ruche et l'impact de l'apiculture sur les autres activités économiques.

Ce travail vise à évaluer la production du miel, son importance économique et médicinale dans l'arrondissement de Manigri.

\section{MATERIEL ET METHODE D'ETUDE Milieu d'étude}

L'étude a été réalisée dans l'arrondissement de Manigri, situé dans la commune de Bassila en République du Bénin (figure 1).

Le climat est de type soudano-guinéen à deux saisons dont une saison de pluies de mi-avril à mi-octobre, et une saison sèche, le reste de l'année (Akoègninou et al., 2006). Le total pluviométrique annuel est en moyenne de $1206 \mathrm{~mm}$. La hauteur moyenne mensuelle d'eau varie de $7,1 \mathrm{~mm}$ en janvier à $223 \mathrm{~mm}$ en août. La moyenne mensuelle de la température oscille entre $24,29^{\circ} \mathrm{C}$ en juillet et $28,88^{\circ} \mathrm{C}$ en mars. Les températures minimales mensuelles sont comprises entre $17,66{ }^{\circ} \mathrm{C}$ en janvier et $22,46{ }^{\circ} \mathrm{C}$ en avril. Les températures maximales mensuelles varient de $28,17{ }^{\circ} \mathrm{C}$ en juillet à 35,90 ${ }^{\circ} \mathrm{C}$ en mars. Les sols sont de types ferrugineux tropicaux (Faure, 1977; Dubroeucq, 1977).

Sur le plan phytogéographique, Manigri appartient à la zone de transition soudano-guinéenne où la végétation est une mosaïque de savanes et de forêt claire parsemée de forêt galerie, de champs et de plantations (Akoègninou et al., 2006).

La population de Manigri est estimée à 16688 habitants (Anonyme, 2003). Elle est dominée par le groupe ethnique Nago. Les activités pratiquées par cette population, par ordre d'importance, sont: l'agriculture, l'élevage de volailles et de petits ruminants, l'apiculture, le commerce, la pêche et l'exploitation forestière (Houinato, 2001).

\section{Méthode}

Des enquêtes sont menées auprès de 35 apiculteurs de l'arrondissement de Manigri, situé dans la commune de Bassila (Département de la Donga). Elles sont réalisées à l'aide de questionnaires semistructurés. Les interviews ont lieu de façon individuelle et en "focus group" de 10 apiculteurs. Le "focus group" permet d'enrichir les informations recueillies par interview individuelle (Lebel et al., 2002). Il est réalisé à l'aide de discussions semidirigées et fait usage d'une check-list de questions guides flexibles où de nouvelles questions ou pistes d'interrogations émergent tout au long de l'entretien (Tamboura et al., 1998).

Les différentes rubriques du questionnaire sont :

- la structure organisationnelle des apiculteurs,

- la connaissance de la pratique apicole,

- les contraintes et atouts financiers de la pratique apicole,

- les usages thérapeutiques du miel.

Les données collectées ont été codifiées pour une analyse fréquentielle avec le logiciel Microsoft office Excel 2003. Pour un paramètre donné, sa fréquence est le rapport exprimé en pourcentage du nombre d'apiculteurs ayant reconnu ce paramètre par le nombre total d'apiculteurs enquêtés.

La rentabilité financière de la production de miel a été évaluée à l'aide de la marge nette de la production en utilisant la méthode coûts/recettes (Dieye et al., 2002 ; 


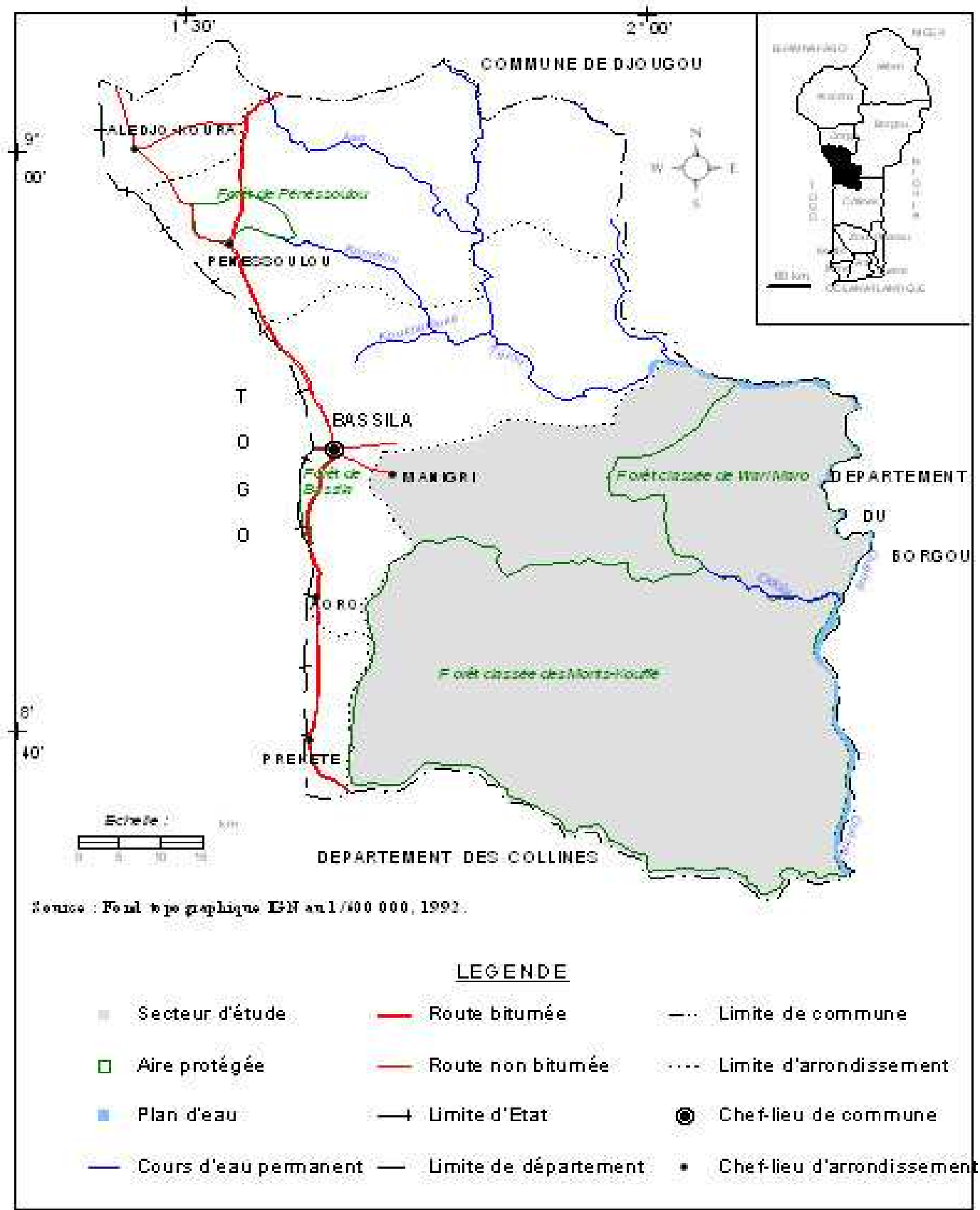

Figure 1 : Localisation de l'arrondissement de Manigri dans la commune de Bassila.

Tossou, 2005). Les coûts ou dépenses totales de production ont concerné les matériels, la main d'œuvre et les dépenses diverses; la main d'œuvre familiale est valorisée au prix du marché. Les recettes brutes ont été estimées à partir des flux monétaires issus de la vente du miel. Les marges nettes ou recettes nettes de la production de miel au terme de la première année d'activité ont été déterminées en déduisant les coûts des recettes brutes.

La nature et le degré de corrélation entre le nombre de ruches exploitées et les variables telles que le volume de miel récolté, les recettes brutes, les dépenses effectuées et la recette nette d'exploitation sont déterminés par la corrélation de Pearson qui est un test 
paramétrique. La normalité des données a été toujours vérifiée au préalable. Le coefficient de corrélation (r) est calculé à l'aide du logiciel MINITAB 13.20. Le test de signification de $\mathrm{r}$ est fait à base du tableau des valeurs critiques. Les valeurs critiques dans ce cas ( $n=35$ apiculteurs enquêtés ou degré de liberté $\mathrm{n}-2=33$ ) sont de 0,335 au seuil de probabilité $\mathrm{p}<0,05$ et de 0,431 à $\mathrm{p}<0,01$.

\section{RESULTATS}

Caractéristiques ethnologiques, structurales et organisationnelles des apiculteurs

Les apiculteurs enquêtés ont un âge moyen de $50 \pm 13$ ans (tableau 1). La tranche d'âge de 30-39 ans renferme le plus fort effectif avec $31,4 \%$ des enquêtés. Elle est suivie de celles de 40-49 ans $(25,7 \%)$ et de 50-59 ans (20\%). Les tranches de 60-69 ans et de 70-79 ans comptent chacune $11,4 \%$. Les jeunes de moins de 30 ans ne pratiquent pas cette activité.

Parmi les personnes enquêtées, 34 soit $97,1 \%$ appartiennent à l'ethnie Nago dont 1 femme (soit 2,9\%). Un seul apiculteur $(2,9 \%)$ est de l'ethnie Mahi. De ces personnes, 48,6\% ne savent ni lire, ni écrire. Les autres sont du niveau primaire $(25,7 \%)$ ou secondaire $(25,7 \%)$.

L'échantillon enquêté est constitué uniquement d'apiculteurs dont l'activité primaire est l'agriculture. Soixante dix pourcent $(70 \%)$ d'eux pratiquaient la chasse au miel comme activité secondaire avant d'être formés pour l'apiculture moderne. Les formations et/ou les recyclages ont été assurés pour $93,6 \%$ des apiculteurs enquêtés par l'ONG Born Fonden en 1999 et le Projet d'Aménagement des Massifs Forestiers (PAMF) en 2006. Ces formations leur ont permis d'avoir de connaissances modernes pour la pratique de l'apiculture. C'est ainsi que $100 \%$ des apiculteurs reconnaissent la plupart des différents groupes fonctionnels de la colonie en dehors des couvains (œufs et larves) reconnus seulement par 9,4\%. Les produits fabriqués par les abeilles (miel, cire, propolis) sont diversement connus: $100 \%$ pour le miel, $81 \%$ pour la cire et $66 \%$ pour la propolis. Ces apiculteurs maîtrisent assez bien la manipulation des ruches et affirment que leur entretien est très capital pour les abeilles. Ils sont, à $100 \%$, conscients des conséquences de la cueillette du miel dans les troncs d'arbres et les termitières avec l'utilisation du feu et l'abattage des arbres sur l'environnement et la biodiversité.

Le piégeage des essaims d'abeilles s'opère à des périodes variées. Pour certains apiculteurs $(65,6 \%)$, il a lieu en saison sèche (octobre à avril); pour d'autres, c'est soit durant la saison pluvieuse $(6,3 \%)$, soit toute l'année $(28,1 \%)$.

S'agissant de la récolte de miel, 53\% des apiculteurs font seulement une récolte par an, durant la période allant de fin février à mai. Les $47 \%$ restants opèrent une seconde récolte entre juillet et septembre, quand les colonies sont fortes.

Parmi les apiculteurs enquêtés, 74,3\% appartiennent à 6 groupements composés de 6 à 20 membres. Les noms en dialecte Nago, tels que "Egui Lowo" (l'arbre c'est de l'argent), "Ifè Lagbara" (l'amour est l'argent), "Iré dé" (le bonheur est arrivé), "Kani Sourou" (il faut avoir la patience et "Ognin don" (le miel est doux), attribués à ces groupements traduisent bien l'importance économique et culturelle du miel.

Tableau 1: Structure des âges des apiculteurs de Manigri.

\begin{tabular}{lcc}
\hline Classe d'âge & Effectif & Pourcentage \\
\hline $0-29$ ans & 0 & 0,00 \\
$30-39$ ans & 11 & 31,43 \\
$40-49$ ans & 9 & 25,71 \\
$50-59$ ans & 7 & 20,00 \\
$60-69$ ans & 4 & 11,43 \\
$70-79$ ans & 4 & 11,43 \\
\hline Total & 35 & 100,00 \\
\hline
\end{tabular}




\section{Importance économique de la production du miel}

Le nombre de ruches installées varie de 3 à 38 avec une moyenne de $11 \pm 17$ (tableau 2). La quantité de miel récolté est en moyenne de 125,2 \pm 95,7 litres par apiculteur. Le prix de vente du litre de miel est compris entre $1000 \mathrm{~F}$ et $1500 \mathrm{~F}$ suivant la période de l'année. Le taux de commercialisation est de $90 \%$ en moyenne et les recettes brutes générées s'élèvent en moyenne à 155000 F/an/apiculteur. Les dépenses renouvelables, relatives à la main d'œuvre et à l'achat de bouteilles sont comprises entre 25630 et 71 $910 \mathrm{~F}$ avec une moyenne de $35280 \pm 8615$ F/an/apiculteur. L'équipement durable (ruches, accoutrements, brosse, bottes, enfumoir et bidons) coûte entre 76720 et 517 280 , avec une moyenne de $180280 \pm 91130$ F par apiculteur.

La figure 2 montre que les facteurs tels que le volume de miel récolté, les dépenses et la recette brute ont une relation linéaire hautement significative avec le nombre de ruches colonisées. Le coefficient de corrélation de Pearson (r) est de 0,89 pour le nombre de ruches colonisées et le volume de miel récolté au seuil de $\mathrm{p}=0,000$ (figure $2 \mathrm{a}$ ). L'équation de droite de régression permettant de prédire le volume de miel $(\mathrm{y})$ à partir du nombre de ruches colonisées (x) est: $\mathrm{y}=$ $11,75 \mathrm{x}-7,27$.

Le coefficient de corrélation de Pearson est de 0,90 pour le nombre de ruches colonisées (x) et les recettes brutes (y) au seuil de $p=0,000$ (figure $2 b$ ). L'équation de la droite de régression entre ces deux variables est : $\mathrm{y}=14856 \mathrm{x}-12458$.

Le coefficient de corrélation de Pearson est de 0,99 pour le nombre de ruches colonisées (x) et les dépenses totales (y) au seuil de $\mathrm{p}=0,000$ (figure $2 \mathrm{c}$ ). L'équation de la droite de régression est y = 13527x-63071.

En considérant la première année d'installation et d'exploitation des ruchers, les coûts totaux de la production de miel s'élèvent de $102350 \mathrm{~F}$ à $589190 \mathrm{~F}$ avec une moyenne de $215560 \pm 98995 \mathrm{~F}$ par apiculteur. Les recettes nettes par apiculteur varient alors de 170800 à $81130 \mathrm{~F}$. Chez la plupart des apiculteurs, les dépenses sont supérieures aux recettes brutes (figure 3). La figure 3 indique aussi la variabilité des recettes nettes au sein des apiculteurs ayant le même nombre de ruches. C'est ainsi que parmi les 5 apiculteurs disposant de 7 ruches, un seul réalise un bénéfice de $5720 \mathrm{~F}$ pendant que les 4 autres connaissent des déficits de 19920 à $72060 \mathrm{~F}$. Il en est de même pour les 3 apiculteurs ayant 12 ruches où l'un a obtenu un bénéfice de 11 $590 \mathrm{~F}$ pendant que les deux autres ont des manques à gagner de 60185 et $115600 \mathrm{~F}$. Aucune corrélation linéaire significative $(\mathrm{p}>$ $0,05)$ n'existe entre le nombre de ruches colonisées et les recettes nettes par apiculteur (figure 4). La courbe de tendance qui permet de mieux prévoir la recette nette, issue de la production (y) en fonction du nombre de ruches (x), est de type polynomial. Son équation est : $\mathrm{y}=282,17 \mathrm{x}^{2}-9019,4 \mathrm{x}-9348,8$. Cette équation permet de prédire seulement avec $30,16 \%$ de succès le bénéfice en fonction du nombre de ruches colonisées.

Tableau 2 : Données statistiques de la production du miel par apiculteur à Manigri.

\begin{tabular}{lc}
\hline Variables & Moyenne \\
\hline Nombre de ruches & $11 \pm 16$ \\
\hline Quantité de miel récolté (litres) par an & $125,2 \pm 95,7$ \\
\hline Prix de vente /litre & $1400 \pm 151$ \\
\hline Recette brute par an & $155000 \pm 120020$ \\
\hline Dépenses renouvelables (bouteilles et mains d'œuvre) par an & $180280 \pm 8615$ \\
\hline $\begin{array}{l}\text { Coût de l'équipement durable (ruches, accoutrements, brosse, } \\
\text { enfumoir, bidon, etc.) }\end{array}$ & $215560 \pm 98995$ \\
\hline $\begin{array}{l}\text { Coûts totaux de production de miel au terme de la première } \\
\text { année d'activité }\end{array}$ & $-60555 \pm 47140$ \\
\hline $\begin{array}{l}\text { Marges nettes de la production au terme de la première année } \\
\text { d'activité }\end{array}$ & \\
\hline \begin{tabular}{l} 
Les chiffres indiquent les moyennes avec leur écart-type, établies sur un nombre (n) de 35 apiculteurs enquêtés. \\
\hline
\end{tabular}
\end{tabular}



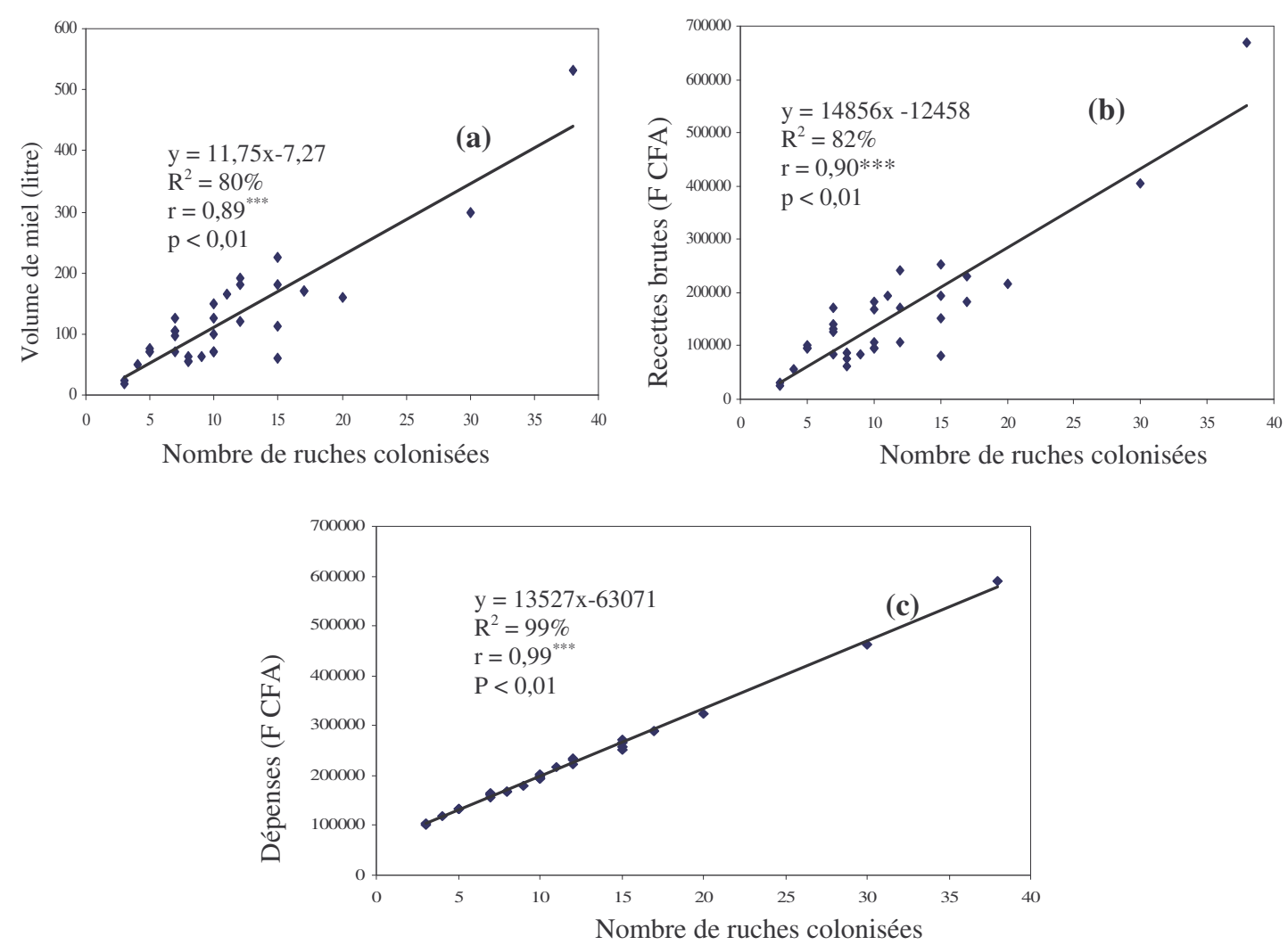

Figure 2 : Relations entre le nombre de ruches colonisées et diverses variables. a : volume de miel récolté $; \mathbf{b}$ : recettes brutes ; $\mathbf{c}$ : dépenses (dépenses renouvelables et dépenses pour l'équipement durable) $; \mathrm{R}^{2}:$ coefficient de détermination; $\mathrm{r}$ : coefficient de corrélation de Pearson; $\mathrm{p}$ : seuil de probabilité ; ***: significatif à $\mathrm{p}<0,01 ;$ (valeur critique de $\mathrm{r}$ pour $\mathrm{ddl}=33$ étant de 0,335 à $\mathrm{p}<0,05$ et de 0,431 à $\mathrm{p}<0,01$ ).

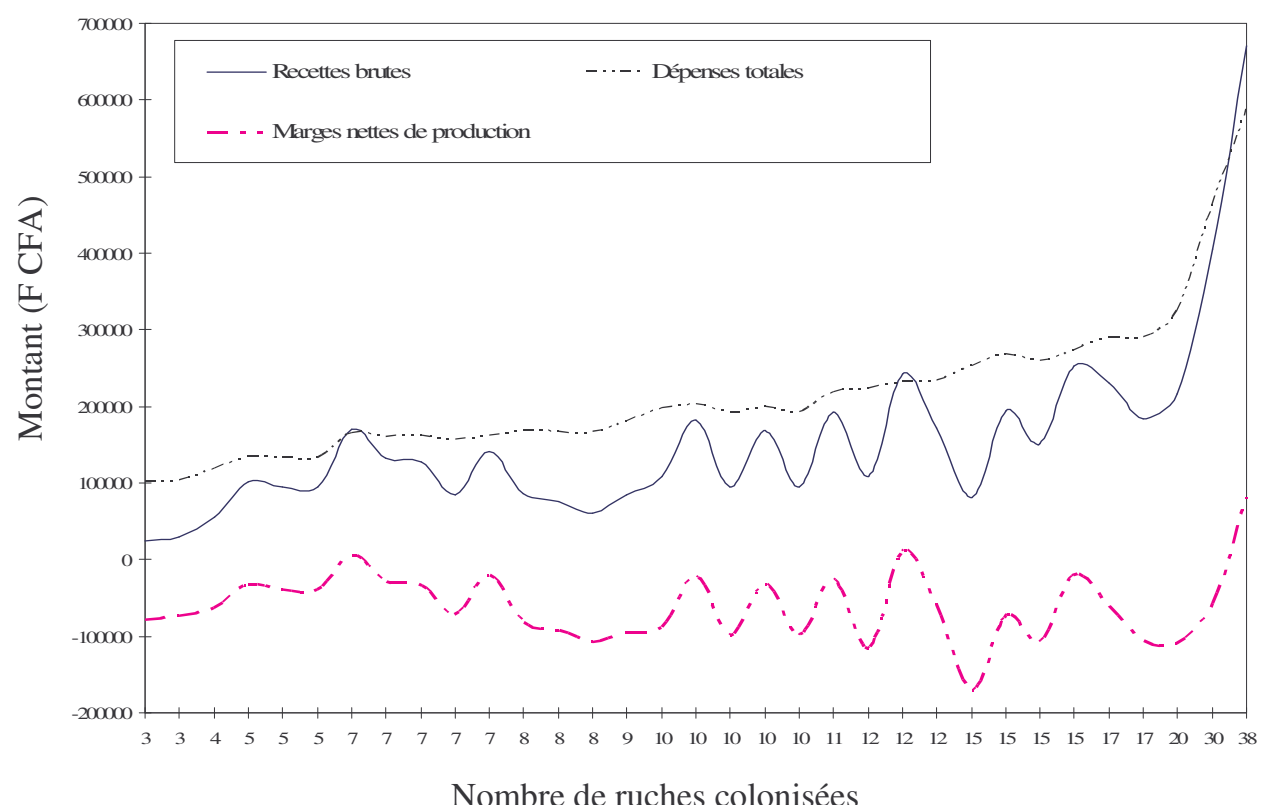

Figure 3 : Evolution des recettes brutes, des dépenses et des marges nettes en fonction du nombre de ruches colonisées, au terme de la première année d'installation et d'exploitation des ruchers. 
Usage du miel en médecine traditionnelle dans l'arrondissement de Manigri

Le miel, en dehors de son utilisation dans l'alimentation et le commerce, présente de nombreuses vertus médicinales. Il est utilisé dans le traitement de 20 affections médico-magiques (tableau 3). La toux et la fièvre sont les maladies pour lesquelles le miel est plus utilisé $(88,6 \%)$. Ensuite viennent, par ordre d'importance, les plaies et les brûlures ( $22,9 \%$ chacune), l'aide mémoire $(17,1 \%)$, les maux de ventre $(14,3 \%)$ et les crises de rhumatisme $(5,7 \%)$. L'utilisation du miel dans le traitement des autres maladies est reconnue seulement par 2,9\% des enquêtés.

Le miel est utilisé à l'état pur dans le traitement de $68,8 \%$ des maladies, sous forme diluée avec une autre solution pour $25 \%$ des affections et en mélange avec des organes de plantes dans $6,3 \%$ des cas.

$\mathrm{Au}$ niveau du mode d'administration des produits, c'est la voie orale qui prédomine à $65 \%$ dont $50 \%$ par lapement et $15 \%$ comme boisson. On rencontre néanmoins d'autres procédés tels que le cataplasme $(20 \%)$ et les instillations oculaire et auriculaire (10\%). De plus, le miel exposé dans une chambre serait porte bonheur.

Quant au rythme d'application du traitement, il est variable en fonction du type et de la gravité de l'affection. Dans l'ensemble, $70 \%$ des prescriptions sont à répéter au moins deux fois par jour et $30 \%$ en application unique pour escompter la guérison.

\section{DISCUSSION}

L'apiculture moderne est pratiquée par les personnes ayant entre 30 et 79 ans à
Manigri. Les jeunes de moins de 30 ans ne la pratiquent pas. Pourtant, la couche juvénile active de 20-29 ans représentent une proportion relativement importante $(14,6 \%)$ de la population de l'arrondissement de Manigri par rapport à celle des apiculteurs (30 à 79 ans) qui est de 24,6\% (Anonyme, 2003). Les jeunes préfèreraient être utilisés comme main d'œuvre ponctuelle pour les travaux d'exploitation forestière. L'extension de l'activité apicole vers cette couche juvénile active constituerait alors un atout important pour son essor. Le nombre d'apiculteurs décroît avec l'âge. Ceci s'expliquerait par le fait que l'apiculture est considérée comme une activité secondaire à l'agriculture et n'est surtout pratiquée que par la couche active des agriculteurs.

La plupart des apiculteurs ont suivi une formation adéquate avant de se lancer dans cette filière. Ils ont acquis les connaissances théoriques et techniques modernes de l'apiculture qui sont des préalables pour une production de miel à grande échelle. En effet, la non maîtrise des techniques apicoles par l'apiculteur conduit aux pertes de colonies d'abeilles (Castagné, 1983 et Nombré, 2003) et, par conséquent, à la diminution sensible de la quantité totale de miel à récolter. La formation reçue par tous les apiculteurs est à mettre à l'actif de l'ONG Born-Fonden et du Projet d'Aménagement des Massifs Forestiers (PAMF). Ces structures ont pour objectif de développer des activités de substitution comme l'apiculture moderne dans les villages riverains des forêts classées d'Agoua, des Monts Kouffé et de Wari Maro (figure 1) afin de réduire l'exploitation intensive des ressources naturelles. Mieux, 74,3\% des

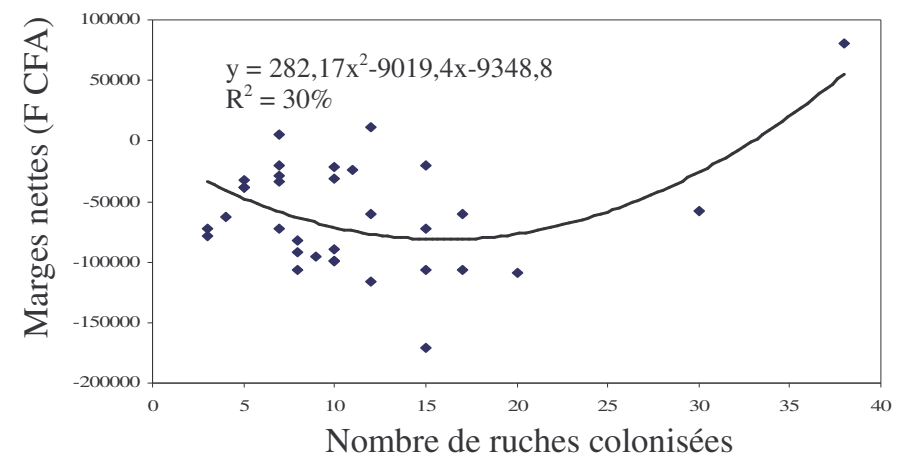

Figure 4 : Corrélation entre le nombre de ruches colonisées et les recettes nettes au terme de la première année d'installation et d'exploitation des ruchers. $\mathrm{R}^{2}$ : coefficient de détermination. 
Tableau 3 : Usages médico-magiques du miel.

\begin{tabular}{|c|c|c|}
\hline Maladie & Mode d'utilisation & Fréq. \\
\hline Toux & $\begin{array}{l}\text { Laper le miel trois fois par jour / boire le miel mélangé au } \\
\text { citron dans une proportion de } 1 \text { volume pour } 2 \text { ou de } \\
\text { l'huile de palme ou de l'éthanol }\end{array}$ & 88,57 \\
\hline Fièvre chez les enfants & Passer le miel sur le corps et le laper & 88,57 \\
\hline Plaies et brûlure & Passer localement le miel sur la partie affectée & 22,85 \\
\hline $\begin{array}{l}\text { Aide mémoire et } \\
\text { intelligence }\end{array}$ & Laper souvent le miel & 17,14 \\
\hline Maux de ventre & Boire le mélange miel + citron & 14,28 \\
\hline Crise de rhumatisme & Laper souvent le miel & 5,71 \\
\hline Courbature & Passer sur le corps & 2,86 \\
\hline Fatigue & Boire le mélange miel + citron + eau & 2,86 \\
\hline Maux d'oreilles & Instiller une goutte de miel dans les oreilles & 2,86 \\
\hline Angine & Boire l'eau tiède au miel & 2,86 \\
\hline Maux de tête & Laper le miel & 2,86 \\
\hline Impuissance sexuelle & $\begin{array}{l}\text { Canne à sucre réduite en poudre + miel + eau à moitié } \\
\text { d'un verre à bière + feuilles de Annona senegalensis }\end{array}$ & 2,86 \\
\hline Maux d'yeux & Instiller deux à trois goûtes de miel dans les yeux & 2,86 \\
\hline Ulcère & Laper de temps en temps le miel & 2,86 \\
\hline Constipation & Prendre le miel dilué dans l'eau & 2,86 \\
\hline Fièvre typhoïde & Laper souvent le miel & 2,86 \\
\hline Maux de gorge & Laper le miel et le passer sur la gorge & 2,86 \\
\hline Dentition chez les enfants & $\begin{array}{l}\text { Prendre le mélange miel + rhizome de chiendent (Imperata } \\
\text { cylindrica) réduit en poudre }\end{array}$ & 2,86 \\
\hline Piqûre de scorpion & Passer le miel sur la partie piquée & 2,86 \\
\hline Effet spirituel & La présence du miel dans la chambre chasse le danger & 2,86 \\
\hline
\end{tabular}

apiculteurs enquêtés appartiennent à des groupements ayant des noms dont les dénominations mettent bien en exergue la valeur économique du miel, de l'arbre et de la filière apicole.

Les volumes de miel récoltés par ruches montrent un bon rendement de miel dans la zone d'étude. Le rendement moyen en miel par ruche, de 11,2 \pm 3,7 litres, est nettement supérieur à celui de 8 à $12 \mathrm{~kg}$ (soit environ 4 à 6 litres) de miel indiqué pour l'Afrique tropicale (Villière, 1987). Il dénote une disponibilité des ressources mellifères et une bonne activité des abeilles dans la zone de Manigri. Ceci confirme que les potentialités de production de miel d'une région sont déterminées par de nombreux facteurs dont la race d'abeilles, le type de ruche, l'environnement et la maîtrise du calendrier apicole (Villières, 1987 ; Nombré, 2003 ; Jansens et al., 2006).

Le nombre moyen de 11 ruches par apiculteur indique que les exploitations apicoles de Manigri sont de petite taille comparativement à celles en Centrafrique où la moyenne est de 40 à 70 ruches par apiculteur (Mbétid-Bessane, 2004). Le volume moyen annuel de miel récolté par apiculteur de Manigri (125,2 \pm 95,7 litres) reste aussi inférieur à celui de 480 litres pour les petits producteurs centraficains ayant 40 ruches et de 840 litres pour les gros producteurs avec 70 ruches (Mbétid-Bessane, 2004). Ces résultats peuvent s'expliquer par le fait que l'apiculture est considérée comme une activité secondaire à Manigri. La spécialisation de ces apiculteurs constitue une nécessité pour accroître la taille des ruchers et mieux maîtriser les facteurs déterminants dans 
la productivité des ruches pour l'optimisation de leurs revenus monétaires annuels.

Le miel produit est surtout destiné à la vente pour l'amélioration des revenus monétaires. Les marges nettes annuelles de la production de miel, par apiculture, varient de $170800 \mathrm{~F}$ à $81130 \mathrm{~F}$ avec une moyenne de $60555 \pm 47140 \mathrm{~F}$. Ceci montre que la plupart des acteurs ne réalisent pas de bénéfice au terme de la première année de production. La raison fondamentale est liée au fait que le coût d'acquisition du matériel d'installation est relativement élevé et n'est pas couvert par les premières recettes brutes. Les dépenses pour l'équipement durable sont de 88000 à $256000 \mathrm{~F}$ pour respectivement 3 et 38 ruches. Elles représentent $90 \%$ du coût total de production au cours de la première année d'installation des ruchers auxquelles il faut ajouter les dépenses renouvelables. Elles semblent être au-delà des possibilités des agriculteurs dont le revenu annuel national en milieu rural varie de 74055 à $125491 \mathrm{~F}$ (Anonyme, 2001). L'autofinancement en apiculture n'est donc pas à la portée des producteurs de miel. Le développement de l'apiculture implique la prise en charge du financement du matériel d'installation par les structures de développement local. Aussi, faudrait-il que ceux qui sont financés puissent investir une partie de leur bénéfice dans les équipements pour augmenter la taille de leur rucher afin d'accroître le volume de miel et par conséquent leur revenu.

La fréquence élevée $(88,6 \%)$ de reconnaissance par les apiculteurs du traitement de la toux et de la fièvre par le miel témoigne de son efficacité pour le traitement. Ceci s'expliquerait par la richesse du miel en de nombreux antibiotiques, regroupés sous le nom générique d'inhibine, qui sont en fait de puissants bactériostatiques (Hakim, 1989; Cortopassi-Laurino et Gelli, 1991). Par rapport aux études sur l'apithérapie au Burkina Faso (Cunchinabé, 1983 ; Guinko et al., 1989; Nacoulma et MillogoRasolodimby, 1995 ; Nombré, 2003), 8 vertus médicinales sont reconnues dans la présente étude. Les 12 restants constituent l'apport du Bénin dans la connaissance des usages médicaux du miel. Ceci serait lié au fait que la médecine traditionnelle reste avant tout un phénomène culturel.

\section{Conclusion}

La zone de Manigri a beaucoup d'atouts pour la production du miel. La production de miel constitue une source potentielle non négligeable de revenus monétaires pour la population rurale, en même temps qu'il peut contribuer à l'amélioration de l'alimentation humaine. L'optimisation des revenus exige que le producteur dispose d'un nombre suffisant de ruches colonisées. Les résultats de cette étude mettent en exergue l'intérêt que les structures de développement local et la population accordent à l'apiculture à Manigri. Ceci implique des moyens financiers d'installation qui ne sont pas à la portée des agro-apiculteurs. L'implication des structures de microfinances est indispensable pour favoriser le développement de cette filière. Le miel est utilisé pour le traitement de diverses maladies.

\section{REMERCIEMENTS}

Les auteurs remercient sincèrement la Fondation Internationale pour la Science (FIS) pour avoir financé cette recherche à travers la bourse B/4014-1.

\section{RÉFÉRENCES}

Akoègninou $\mathrm{A}$, van der Burg WJ, van der Maesen LJG. 2006. Flore Analytique du Bénin. Backhuys Publishers: Wageningen; 1034.

Anonyme 2001. Etude sur les Conditions de Vie des Ménages Ruraux: Profil de Pauvreté Rurale et Caractéristiques Socio-économiques des Ménages Ruraux. MAEP ; 84.

Anonyme 2002. L'utilisation des Produits Forestiers non Ligneux dans le Cadre de la Gestion Forestière Durable. Flamboyant; 56.

Anonyme 2003. Troisième Recensement Général de la Population et de l'Habitation. Direction des Etudes Démographiques. INSAE-UNICEFUNFPA- DDC; 404.

Anonyme 2005. Production Documentaire et Statistique sur le Coton, le Palmier à Huile, le Lait, l'Apiculture, l'Aviculture et le Crédit Rural en Afrique de l'Ouest et du Centre. FAO ; 14.

Castagné JB. 1983. L'apiculture au CongoBrazaville. Bul. Tech. Apic., 10(4): 197208. 
Cortopassi-Laurino M, Gelli DS. 1991. Analyse pollinique, propriétés physicochimiques et action antibactérienne des miels d'abeilles africanisées Apis mellifera et Meliponinés du Brésil. Apidologie, 22: 61-67.

Cunchinabé D. 1986. Apiculture en voie de développement, Burkina Faso. Rev. Franc. Apic.: 26-28.

Dieye PN, Faye A, Seydi M, Cissé SA. 2002. Production laitière périurbaine et amélioration des revenus des petits producteurs en milieu rural au Sénégal. Cahiers Agriculteurs, 11: 251-257.

Dubroeucq D. 1977. Carte pédologique de reconnaissance de la République Populaire du Bénin à 1/200 000 : feuille Parakou. ORSTOM, notice explicative, 66(5) : 37.

Faure P. 1977. Carte pédologique de reconnaissance de la République Populaire du Bénin à 1/200 000 : feuille Djougou. ORSTOM, notice explicative, 66(4): 49.

Guinko S, Guenda W, Millogo-Rasolodimby J, Tamini Z, Zoungrana I. 1989. Apithérapie: quelques usages du miel dans l'Ouest du Burkina Faso. Revue de Médecines et Pharmacopées Africaines, 3(2): 111-115.

Hakim H. 1989. Le miel aliment-médicament. Angéiologue, p. 35.

Houinato M. 2001. Phytosociologie, écologie, production et capacité de charge des formations végétales pâturées dans la région des Monts Kouffé (Bénin). Thèse de Doctorat, Université Libre de Bruxelles, p. 219.

Hussein MH. 2001. L'apiculture en Afrique. Apiacta, 1: 34-48.

Janssens X, Bruneau E, Lebrun P. 2006. Prévision des potentialités de production de miel à l'échelle d'un rucher au moyen d'un système d'information géographique. Apidologie, 37: 351-365.

Lebel F, Debailleul G, Samba SAN, Olivier A. 2002. Contribution des produits forestiers non-ligneux à l'économie des ménages de la région de Thiès, au Sénégal. Acte $2^{\text {ème }}$ Atelier Régional sur les Aspects socio-économiques de l'agroforesterie au Sahel, Bamako 4-6 mars 2002, pp. 1-9.

Mbetid-Bessane E. 2004. Apiculture, source de diversification des revenues des petits agriculteurs: cas du basin cotonnier en Centrafrique. Apicultura, 22(3): 156-158.

Nacoulma O, Millogo-Rasolodimby J. 1995. Les produits de la ruche et leurs utilisations au Burkina Faso. Revue de Médecines et Pharmacopées Africaines, 9(2): 63-70.

Nombré I. 2003. Etudes des potentialités mellifères de deux zones du Burkina Faso : Garango (Province du Bouglou) et Nazinga (Province du Nahouri). Thèse de Doctorat, Université, Burkina Faso., 156 p.

Tamboura H., Kaboré H. \& Yaméogo S. M. 1998. Ethnomédecine vétérinaire et pharmacopée traditionnelle dans le plateau central du Burkina Faso : cas de la province du Passoré. Biotechnol. Agron. Soc. Environ, 2(3) : 181-191.

Tossou A. G. E. 2005. Analyse socioéconomique du système de commercialisation des amendes et du beurre de karité (Vitellaria paradoxa) dans les communes de Kandi et Gogounou. Thèse d'Ingénieur Agronome, FSA/UAC, p. 65.

Villières B. 1987. L'apiculture en Afrique Tropicale, Dossier « Le point sur», 11, GERT : Paris. 